\title{
EL 2011 ESTUDIANTIL CHILENO COMO DESAFÍO ANALÍTICO PARA LAS CIENCIAS SOCIALES: HACIA LA CONSTRUCCIÓN DE UNA NUEVA MATRIZ PARA LEER LOS MOVIMIENTOS (2001-2011 Y MÁS ALLÁ)
}

DANIEL FAURÉ ${ }^{1}$

\begin{abstract}
RESUMEN
El ciclo de movilización estudiantil chileno lleva más de quince años de desarrollo. Sin embargo, el clímax de éste el 2011 sorprendió a las ciencias sociales, quienes tuvieron que efectuar lecturas de emergencia del fenómeno en las que redujeron las movilizaciones a la condición de fuerza social, investigando mayormente la dimensión externa del movimiento (los repertorios de acción en el espacio público, la porosidad del modelo político para procesar demandas, etc.), relegando a un segundo plano su dimensión interna, donde se configuraría el proyecto alternativo. En el artículo, se realiza un balance crítico de estas lecturas de emergencia pos 2011 (en particular de la Nueva Historia Social) y se confrontan con la producción del pensamiento crítico latinoamericano sobre los movimientos sociales, buscando constituir una matriz analítica situada.
\end{abstract}

Palabras ClaVe: MOVIMiento estudiantil, MOVIMiENTo social, NUEVA HISTORIA SOCIAL

1 Doctor en Historia de Chile, Universidad de Chile. Profesor adjunto del Departamento de Historia, Universidad de Santiago de Chile. Correo electrónico: daniel.faure@usach.cl. 


\title{
O ANO 2011 ESTUDANTIL CHILENO COMO DESAFIO ANALÍTICO PARA AS CIÊNCIAS SOCIAIS: RUMO À CONSTRUÇÃO DE UMA NOVA MATRIZ PARA A LEITURA DOS MOVIMENTOS (2001 - 2011 E ALÉM)
}

\begin{abstract}
RESUMO
O ciclo de mobilização estudantil chileno tem mais de quinze anos de desenvolvimento. No entanto, o clímax deste é no ano 2011 e surpreendeu as ciências sociais, quem tiveram que realizar leituras de emergência do fenômeno nas que reduziram as mobilizações ao status de força social, pesquisando principalmente a dimensão externa do movimento (os repertórios de ação no espaço público, a porosidade do modelo político para processar demandas, etc.), relegando a um segundo plano sua dimensão interna, onde o projeto alternativo seria configurado. No artigo, é realizado um balanço crítico destas leituras de emergência após 2011 (em particular da Nova História Social) e são confrontados com a produção do pensamento crítico latino-americano sobre os movimentos sociais, visando construir uma matriz analítica situada.
\end{abstract}

PALAVRAS-CHAVE: MOVIMENTO ESTUDANTIL, MOVIMENTO SOCIAL, NOVA HISTÓRIA SOCIAL.

\section{STUDENT DEMONSTRATIONS AS AN ANALYTICAL CHALLENGE FOR SOCIAL SCIENCES: TOWARDS THE CONSTRUCTION OF A NEW MATRIX FOR READING MOVEMENTS (2001 - 2011 AND ON)}

\begin{abstract}
Although the student demonstration cycle has been developing for more than fifteen years, its climax in 2011 took Social Sciences by surprise. This situation forced them to generate emergency readings, to reducing demonstrations to a condition of social force, focusing, mainly, on the external dimension of the movement (the action repertoires in the public sphere, the porosity of the political system to process demands, etc.), and relegating its internal dimension, where an alternative project would develop, to a secondary place. These post-2011 emergency readings (particularly, from the New Social History, and the production of Latin American critical thinking on the social movements are balanced in the article, in order to establish a contextualized and analytical matrix.
\end{abstract}

KEYWORDS: STUDENT MOVEMENT, SOCIAL MOVEMENT, NEW SOCIAL HISTORY. 


\section{INTRODUCCIÓN: EL 2011 ESTUDIANTIL COMO PARTEAGUAS}

MUCHO SE HA ESCRITO en torno a las movilizaciones estudiantiles que sacudieron Chile el año 2011 al punto de transformar dicho año en un parteaguas sociopolítico, un hito aceptado socialmente que marca un antes y un después de cualquier análisis. Su magnitud y explosividad, no prevista por las ciencias sociales chilenas, las desafió a entregar respuestas rápidas tanto para entender el fenómeno como para guiar la acción histórica del movimiento.

En estas páginas, revisaremos las lecturas de emergencia que realizaron dichas ciencias sociales chilenas y, en particular, la Nueva Historia Social (en adelante NHS) a lo largo de ese año y el siguiente para, a partir de esa muestra temprana, reflexionar sobre las categorías emergentes empleadas para caracterizar y analizar a este sujeto colectivo (con énfasis en la categoría de movimiento social) y las dimensiones del fenómeno más estudiadas, todo bajo el objetivo de construir una matriz analítica de carácter histórico que, situada y fechada, dé cuenta de la novedad histórica que este sujeto plantea.

\section{EL 2011: SUS PRIMEROS ANÁLISIS POR ARRIBA Y POR ABAJO}

El movimiento telúrico-social sacudió y agrietó viejas estructuras, dejando secuelas permanentes: en el plano político-estatal, profundizó la crisis de representatividad de la clase política civil, diagnosticada desde hace casi veinte años (Salazar y Pinto, 1999), y la crisis de legitimidad del modelo político de democracia representativa; en el plano económico, generó fisuras en el modelo neoliberal. Crisis simultáneas, consecuencia de dos cosas: primero, un cuestionamiento profundo al discurso de la meritocracia y del ascenso social individual por vía de la educación (Mayol, 2012, p. 85); y, segundo, por la "politización del malestar", que se incubaba en el seno de las grandes mayorías 
de nuestro país por los excesos del mercado desregulado (Durán, 2011, p.49; Mayol, 2012; Núñez, 2011, p.63).

Pero estas crisis y fisuras estructurales, que contaron con una rápida revisión de las ciencias sociales, estuvieron acompañadas por otros cambios $a$ ras de calle: masificación y validación de la movilización callejera como repertorio de acción y, por consiguiente, una recuperación del espacio público como espacio político (donde se plantean demandas y propuestas de orden social), permitiendo visibilizar demandas ciudadanas hasta hace poco sin tribuna pública y dándole al movimiento estudiantil mayor anchura y espesor social, permitiendo la vinculación simbólica de su ciclo de movilización con las jornadas de protesta nacional desarrolladas durante Dictadura, sobre todo a partir de la protesta del 4 de agosto de 2011 (Fauré, 2011).

Sin embargo, estos cambios callejeros -que interpelan nuestra forma de entender la política y lo público- fueron menos estudiados que los anteriores. A lo sumo, se analizaron las manifestaciones como expresión de "fuerza" que instaló ciertas temáticas en la agenda pública pero que, al corresponder a una fase de "negatividad" de los movimientos sociales (en los que se "identifican problemas"), tendría escasas posibilidades de transformarse en un programa de reforma o "positividad" (Atria, 2012, p.28-29, 103). En la misma línea, al conceptualizar la movilización como "fuerza", se hicieron más preguntas en torno a quién podría "capitalizar y conducir esta fuerza", diagnosticando las divisiones al interior del movimiento que lo impedirían (Durán, 2011) y la ausencia de referentes políticos externos que cumplan con ese rol (Ruiz y Sáez, 2012) evidenciando la necesidad de construir, desde el movimiento, órganos de representación política que lleven sus intereses al sistema político existente.

El análisis anterior llevó finalmente a que el foco de atención saliera de la fuerza callejera concentrándose en la esfera político-estatal, con sendos estudios que desenmascararon la (in)capacidad del modelo político chileno para procesar las demandas sociales, lo que se acrecentaba en el gobierno piñerista 
(Tironi, 2011), por obra y gracia del marco institucional, nuestra "jaula de hierro" weberiana (Ramis, 2009) heredada por la clase política civil de la Dictadura (Núñez, 2011, p.64), lo que nos condenó a que cada conflicto termine en una especie de "veto mutuo" (Ruiz y Sáez, 2012, p.37) o "empate catastrófico" (Garcés, 2012, p.20) entre los y las movilizadas y la clase política.

Pero si esta última dimensión del fenómeno fue escasamente estudiada (a no ser que sea en relación al sistema político-institucional), hay otra que pasó desapercibida: la dimensión interna del movimiento. En esta línea, son pocas las reflexiones que se pueden referenciar (todas pre 2011). Así, el Observatorio de Políticas Educativas de Chile (OPECH, 2010), centró la mirada, primero, en las innovaciones en las dinámicas asociativas y organizativas dentro del movimiento estudiantil secundario, con énfasis en el ciclo de movilizaciones 2006-2008, donde se afianzó la dinámica asamblearia como respuesta a la crisis de representatividad antes mencionada; y, segundo, levantó innovadoras hipótesis en torno al rol creciente de la autoeducación en el desarrollo y proyección del movimiento y en la "construcción de ciudadanía". Práctica llevada a cabo en paralelo, pero interconectada, con un movimiento juvenil de "auto-educación popular", de compleja cartografía, situado en los límites y fuera del sistema formal de educación (Fauré, 2012; González, 2009). Similar reflexión hizo Álvarez (2008), quien analizó el asambleísmo secundario como proceso histórico (presente al menos desde el 2000), constituyéndose en un "ideal" a conseguir por los estudiantes, pero que se levanta sobre un acumulado experiencial que proviene de los horizontales colectivos estudiantiles o "piños", que conformarían los nudos que componen la red espesa que sostiene al movimiento estudiantil secundario.

De esos estudios pioneros, poco se avanzó post 2011: sólo $\mathrm{OPECH}$ (González y Sánchez, 2012) avanzó en una mirada global desde "El mochilazo" del año 2001 en adelante, en lo que denominaron "una década de lucha por autoeducación", ciclo cuyo fruto fundamental serían incipientes propuestas de 
"autogestión educativa", las que fueron recogidas -solo testimonialmente- en Trazas de Utopía, obra que recorre los derroteros de los cuatro liceos que, durante el 2011, tuvieron un lapsus de funcionamiento auto gestionado por sus comunidades educativas (Colectivo Diatriba, OPECH y Centro Alerta, 2011). Así, esta dimensión interna del proceso sigue estando sub estudiada, (a pesar de lo provocador de las hipótesis que surgen) y el acervo de información que se está acumulando, clama revisión.

Con todo, un primer balance de los estudios emergentes sobre las movilizaciones en torno a la educación deja un resultado dispar, con preponderancia en el análisis de los modelos por sobre el de los sujetos: con preponderancia en lo político-estatal (ya sea en la conformación de nuestro modelo de modernización autoritaria, en la porosidad del modelo para procesar las demandas ciudadanas o en las fisuras y contradicciones que se abren en ambos), reduciendo el análisis de la acción colectiva a sus formas visibles (los repertorios de acción que se despliegan en el espacio público y las dinámicas de negociación y lucha con sus oponentes) y postergando una lectura interna del fenómeno.

Caso similar ocurre cuando revisamos las categorías con las que se nombra al sujeto movilizado, donde se percibe una tendencia al traspaso de la historicidad desde el sujeto a la institucionalidad: al revisitar a los autores reseñados, se percibe que aquellos que bautizan al sujeto (o su accionar) con categorías como "estudiantes movilizados”, "revuelta estudiantil” (Ruiz y Sáez) o "movimiento estudiantil" (Mayol), tienden a leer el ciclo de movilización de éstos como parte de un guion establecido -y aceptado-, en tanto actor social reconocido e integrado a la dinámica política institucional moderna. De igual manera, aquellos que bautizan al sujeto en movimiento como movimiento social (Durán, Atria, Núñez), en términos generales, limitan el accionar de éstos a su capacidad de articular y visibilizar demandas parciales que deben ser procesadas por la sociedad política y donde, por lo mismo, el éxito del 
movimiento se basa en la capacidad de ganar dicha contienda ya sea movilizando inteligentemente sus recursos, aprovechando coyunturas $\mathrm{u}$ oportunidades políticas o generando alianzas dentro y fuera del aparato estatal. Tendrían, por ello, una vida "corta y luminosa" (Atria, 2012, p.16) a menos que enfrenten, seriamente, el problema de la representación.

En paralelo, en la vereda de los que han dado más énfasis a la dimensión interna, se tiende a la dispersión conceptual. Conviven, a lo largo de los escritos, categorías como movimiento popular o con un fuerte componente popular -sobre todo en las movilizaciones secundarias- (OPECH, 2010) o indistintamente los de movimiento juvenil, movimiento estudiantil y movimiento social por la educación (González y Sánchez, 2012).

Lo anterior abre desafíos a las ciencias sociales que creemos importante encarar en investigaciones futuras. El primero, está en nivelar la cancha de la investigación abriéndose al estudio sistemático de la dimensión interna del sujeto en movimiento. No sólo por la necesidad de comprender globalmente el fenómeno, sino por lo que se anuncia: si el rápido balance de las investigaciones hechas en esta área nos muestran una tendencia a ver acá una capacidad proyectiva, autogestiva y comunitaria (que se oculta al mirar sólo la dimensión externa), urge un análisis que rescate esta capacidad propositiva y sistematice lo elaborado para fortalecer dichas propuestas sociales que superen la crisis educativa aún sin solución. Un desafío disciplinar pero, sobre todo, político y de futuro.

Lo anterior, implica un giro en la forma de concebir los movimientos. Asumiendo, por ahora, que las definiciones dadas de movimiento social los entienden como fenómenos temporalmente acotados, definidos por demandas parciales y con la mirada fija en la sociedad política; el análisis de su dimensión interna podría mostrar que esta fase visible -en el espacio público- no sería sino la punta del iceberg de un fenómeno más profundo. De ser así, las acciones colectivas que se desarrollan en esta profundidad serían de igual o mayor 
importancia para entender la constitución del sujeto social, sus códigos para interpretar su período histórico, sus repertorios de acción y su proyección, que el análisis de su faceta pública. Además, permitiría lecturas de mediano y largo alcance que iluminen las zonas de formación y latencia de estos movimientos, donde se fraguan parte importante de estas futuras acciones públicas.

El segundo desafío, derivado del anterior, es construir las categorías que nos permitan leer a este sujeto en movimiento en sus dos dimensiones. Aquí, si bien movimiento social parece llevar ventaja, es necesario reconocer que éste es un concepto no procesado con profundidad por las ciencias sociales en sus lecturas de emergencia, y desconectado o retrasado -diez años, al menosde los debates latinoamericanos, los que miran con recelo esta categoría por su origen europeo, en tanto fue pensada para la realidad del norte industrializado de los 60 y recepcionada acríticamente por nuestras universidades (Zibechi, 2011a, p.67-68).

El tercer desafío es definir el aporte que la disciplina histórica puede tener en la resolución de los dos primeros. Colocado ahora el movimiento en un plano temporal mayor, y llevado desde el espacio público al interno (y viceversa), creemos que la disciplina histórica, en general, y la NHS, en particular, tiene un importante rol que jugar tanto por el aporte que podemos hacer en el análisis de las continuidades y rupturas de la dualidad movimientolatencia en el plano temporal, como por el acumulado de experiencias investigativas construidas desde una concepción dialógica -desde los sujetos-, experiencias que, claramente, pueden facilitar el análisis de estos períodos de latencia, los más esquivos hasta ahora para nuestras ciencias sociales. Todo esto tras el objetivo no de jerarquizar entre las dimensiones en que se manifiesta el proceso histórico (a fin de cuentas, estas divisiones son analíticas y en la cotidianeidad se presentan fusionadas), sino por la necesidad, en esta etapa, de construir un marco interpretativo general que complemente, no que aísle 
dimensiones y que pueda servir de insumo para los mismos sujetos en movimiento.

Lo último, plantea la necesidad de revisar lo que hemos avanzando como disciplina y como corriente dentro de ella. Reconociendo que la NHS tuvo un papel importante en los debates intradisciplinarios y públicos abiertos en torno al ciclo de movilizaciones, se abre una oportunidad para realizar una cartografia de nuestros gerundios interpretativos tanto para diagnosticar de qué forma estamos nombrando esta realidad (las categorías empleadas) como para dilucidar qué dimensiones del fenómeno están siendo observadas y valoradas.

\section{DEL TRIUNFALISMO HISTÓRICO, LA POLÍTICA 'REALMENTE EXISTENTE' Y LA 'CULTURA SOCIAL'}

Comenzaremos esta sección con una pequeña provocación. En el trabajo de Ruiz y Sáez (2012), los sociólogos chilenos intentan construir una interpretación que nos explique porqué la generación de los "hijos de la modernización" -autoritaria- (p.28) había decidido patear el tablero a punta de movilizaciones, desde el 2006. Su texto comienza con un párrafo sugerente donde se quiere mostrar la importancia de la temática, que ya ha sido destacada -al parecer, de sobra- por otros investigadores y personajes políticos. Para ello, declaran:

Mucha tinta se ha gastado en señalar que el 2011 marca un antes y un después en el decurso histórico reciente de Chile. Se ha hablado de un cambio en las formas de entender la política, de un 'nuevo amanecer de los movimientos sociales', de 'la primavera chilena' e incluso, entre los más entusiastas, de una 'coyuntura pre revolucionaria'”. (p.29)

El gesto es sutil, pero interesante, ya que -en el resto de su artículoconstruyen una lectura alejada de esta especie de triunfalismo inicial bautizando 
al sujeto y al proceso, como señalamos anteriormente, con categorías como “estudiantes movilizados" o "revuelta estudiantil", alejado de "amaneceres", "primaveras" y "coyunturas pre-revolucionarias”. Ahora, ¿por qué tanto revuelo por una cita? Porque, precisamente, este "triunfalismo" proviene, en parte importante, de la misma NHS, en tanto las frases citadas son de Sergio Grez, Ricardo Lagos y Gabriel Salazar, respectivamente.

¿Qué es lo que diferencia al análisis sociológico del histórico en torno a este tema? ¿Estamos hablando sólo de diferencia en las nomenclaturas o ambas disciplinas están viendo procesos -o dimensiones- diferentes? Para contestar ello, revisaremos los postulados de tres historiadores que levantaron las primeras lecturas de emergencia sobre el fenómeno del 2011 -Sergio Grez, Mario Garcés y Gabriel Salazar-, en tanto representantes destacados de la historiografía nacional y, en particular, de la NHS.

\section{SERGIO GREZ Y EL MOVIMIENTO POR LA EDUCACIÓN PÚBLICA}

Grez realizó variadas apariciones en prensa -escrita y televisivadurante el ciclo de movilizaciones del 2011, aportando también en espacios auto-educativos dentro y fuera del ámbito universitario. Dentro de esas apariciones, quiero destacar dos. En su artículo Un nuevo amanecer de los movimientos sociales (2011), Grez divide su análisis en dos partes. Por un lado, explica la coyuntura del 2011 a partir de lo que denomina el "aletargamiento" que centra, fundamentalmente, desde los inicios de la transición. Un ciclo de mediano plazo donde el efecto adormecedor de la ciudadanía estaría dado por la combinación de cinco elementos:

(...) la acción "natural" del modelo económico neoliberal, del recuerdo del régimen de terror de la dictadura, de las trabas y cortapisas legales e institucionales para la expresión de las demandas sociales, de la 
virtual dictadura mediática impuesta por un puñado de grupos económicos y de poder, además del control y cooptación de estos movimientos ejercidos durante largo tiempo por los gobiernos de la Concertación y sus partidos. (Grez, 2011)

Lo anterior, habría generado una crisis de legitimidad doble, tanto del modelo económico neoliberal como del sistema de democracia restringida, tutelada y de baja intensidad sobre la que aparece y se monta, crítico, el sujeto colectivo. En ese contexto se gestó una dinámica organizativa que responde, con hechos, a dicha crisis doble -aunque sobre todo a la política-:

Al mismo tiempo se extiende el cuestionamiento a las viejas formas "delegadas" de hacer política a través de representaciones institucionales divorciadas de las bases sociales, altamente centralizadas y jerárquicas. En su lugar, los jóvenes y otros actores sociales han venido construyendo desde hace años formas más democráticas y horizontales (...) El sistema político binominal, la elitización de la política "profesional" y los abusos de la "clase política", han engendrado sus propios sepultureros: una ciudadanía popular y de clases medias crecientemente empoderada. (Ibíd.)

Eso sería la base sobre la cual se hace visible el sujeto colectivo del 2011, un "movimiento social" amplio donde la "columna vertebral y principal componente son los estudiantes", pero que agrupa a otros sectores en lo que Grez denomina el "movimiento por la educación pública” (Ídem). Hasta acá, la reflexión de Grez -parafraseando a Pérez Ledesma (1994)- ${ }^{2}$ logra situar muy

\footnotetext{
${ }^{2}$ Este autor, en un trabajo de 1994, desarrolla un estado del arte de las ciencias sociales europeas y norteamericanas en torno al concepto de "movimiento social". Su argumentación muestra cómo se ha generado, hacia la década del '90, una división entre una tradición norteamericana, más pragmática, que se ha interrogado sobre el cómo de la "acción colectiva"; y una tradición europea que se ha ocupado de los porqués de los movimientos. Esta diferencia entre porqués y cómos nos parece útil en tanto complementa la división en dimensiones (interna y externa) que habíamos planteado anteriormente. Así, podemos tener, al mismo tiempo, una dimensión externa enfocada en los porqués (causas estructurales y superestructurales del
} 
bien el porqué del movimiento. Sin embargo, este porqué refiere más a lo externo que a lo interno, en tanto no queda claro cómo estos sujetos, en pleno "aletargamiento" han conseguido constituir dinámicas asociativas y organizativas por fuera de la representación estatal (o, derechamente, contra ella). Por ello, la complejidad del análisis de Grez es precisamente la apuesta a futuro que realiza a partir de esta lectura. Para él, en tanto los movimientos sociales se orientan por reivindicaciones sectoriales y corporativas -como sería el caso del movimiento por la educación pública-, corren el riesgo de caer en la "anomia política" y estancarse en un interminable derrotero de "estallidos sociales". Para evitarlo, según Grez, se necesitaría dar el paso siguiente: elaborar propuestas políticas propias conformando plataformas unitarias y democráticas, al tiempo que generan representaciones políticas propias. Frente a ello, cabría preguntarse si es tan fácil resolver el problema de la representación en tanto una parte importante de las orgánicas que se han levantado en este último tiempo -y que recoge Grez en su artículo- se han conformado rehuyendo de dicha representación.

Con todo, Grez construye su definición de movimiento social en un formato clásico: como agrupaciones que en el seno de la sociedad civil enarbolan demandas no procesadas en la sociedad política y que, a partir de su movimiento, re politizan esta misma sociedad civil, socializando la discusión política en diversos espacios sociales, generando correlaciones de fuerzas favorables para, vía representación en el plano político-institucional, procesar dichas demandas. Esto aparece con claridad cuando critica las tesis "maximalistas e intransigentes", representadas por Gabriel Salazar y que operarían en ciertos sectores estudiantiles, que podrían llevar al "desarme

movimiento) y otra centrada en los cómos (repertorios de acción para enfrentar esas causas externas). De la misma manera, es posible considerar un análisis de los porqués de la dimensión interna (los códigos con los que procesan los sujetos el condicionamiento estructural y superestructural, los horizontes de acción que construyen) y un análisis de los cómos de la dimensión interna (repertorios de acción que impliquen construcción del sujeto colectivo: identidad, memoria, cultura social, autoeducación, etc.). Ver: Pérez Ledesma, 1994, pp. 51-120. 
ideológico y político de los movimientos sociales contestatarios”. Acá, Grez hace un llamado a no dar la espalda a:

(...) las mediaciones y conflictos de la política realmente existente, ignorando al Estado y las correlaciones de fuerza entre los actores sociales y políticos (...) un espejismo que sólo puede sembrar derrotas y generar impotencia entre sus seguidores. Su único horizonte es la esterilidad política y el cultivo de una eterna rebeldía que no puede transformarse en poder efectivo. Para evitar ese callejón sin salida, conservando su autonomía, los movimientos sociales pueden y deben abrirse al juego de la política, procurando generar sus propios instrumentos políticos so pena de verse obligados a retirarse a las áridas tierras de la Utopía fundamentalista o a delegar en otros la representación de sus intereses. (Grez, 2012, p.7)

\section{MARIO GARCÉS Y EL DESPERTAR DE LOS MOVIMIENTOS SOCIALES}

Este historiador y educador popular aportó a las lecturas emergentes con un trabajo titulado El despertar de la sociedad. Los movimientos sociales en América Latina y Chile (2012), el cual contenía un análisis en torno al ciclo de movilización reciente. El libro, socializado en espacios autoeducativos, contiene las tesis que luego Garcés replicó en diversos medios de prensa, fundamentalmente alternativos, en los años posteriores.

Para el autor, el fenómeno que estamos aun viviendo estaría protagonizado por un doble "movimiento social”: “(...) los estudiantes, que en rigor, podríamos definir como movimiento social, en sentido estricto; y, la del apoyo de la sociedad, que podríamos definir como un movimiento social en sentido amplio" (Garcés, 2012, p.9). Movimiento que, a su vez, estaría definido como: 
(...) una acción colectiva que se constituye desde la sociedad civil, o desde lo social, para hacer visible el malestar y diversas demandas al Estado y sus instituciones y representantes, o a un oponente en la propia sociedad civil (los patrones, por ejemplo, en el caso del sindicalismo). Lo propio de los movimientos sociales es la acción colectiva de quienes buscan expresar al conjunto de la sociedad su malestar y sus proyectos de cambio social. (p.10)

Acá, el autor abre una brecha interesante, en tanto señala que dicha acción no necesariamente debe estar dirigida al Estado y sus instituciones sino que dentro de la misma sociedad civil. Así, lo definitorio sería la capacidad de este sujeto colectivo de "ponerse en movimiento".

Pero dejemos esto en pausa para ver otros elementos clave en su análisis. Para Garcés, el porqué externo del surgimiento de este doble "movimiento social" viene dado por el corsé institucional que se generó con la Constitución del 80 -escasamente modificado por los gobiernos pos dictatoriales-, que inhibe la democracia en aras de la estabilidad (Garcés, 2012, pp.16-17). Así, la congruencia con el análisis de Grez en este punto es grande, adscribiendo también a la doble crisis antes referida: "crisis de credibilidad" -le dirá Garcés- que, expresada con mayor fuerza en el primer gobierno de Piñera, no sería sino la superficie de una "crisis de legitimidad" que la subyace. En esa línea, el callejón sin salida es precisamente el señalado antes: las vías de salida al conflicto que, esta vez, sería entre el movimiento y la "clase política". ¿Dónde se pueden dirimir las diferencias? Garcés señala:

La respuesta sería en el sistema político vigente, o sea, en las instituciones, ya sea porque se llega a acuerdo con el ejecutivo o porque se modifican las leyes en el Parlamento. Pero aquí volvemos al problema inicial: el sistema político chileno, organizado sobre la base de una Constitución heredada de la Dictadura, obliga a altas mayorías parlamentarias para denominar las "leyes orgánicas" (la de educación 
es una de ellas), lo que supone un acuerdo con la derecha neoliberal -la trampa a la que se acomodó la Concertación por 20 años en el Gobierno-, pero además la Constitución tampoco hace posible convocar a un plebiscito. (p.20)

Así, estaríamos en presencia de una situación límite en tanto, frente a la incapacidad de procesar la demanda por la vía de la política "realmente existente" de Grez, la otra política (la de la movilización) no haría sino equiparar la primera, sin superarla. Prosigue Garcés:

Nos acercamos de este modo a un "empate catastrófico" en que el Gobierno cuenta con todos los medios institucionales para bloquear al movimiento estudiantil y apostar a su desgaste en el tiempo, y los estudiantes están obligados a mantenerse movilizados y sumar apoyos sociales para no perder visibilidad y lograr reformas significativas en el actual sistema educativo nacional, o contribuir a que la crisis de legitimidad se transforme en crisis política. O sea, que obligue al ejecutivo y al Parlamento a modificar el sistema político para hacer posible procesar las demandas que provienen de los movimientos sociales. (p.21)

Ahora, a pesar de lo interesante de la propuesta, Garcés no ahonda en esta línea. Sin embargo, si lo hace en el porqué interno, inscribiendo a los movimientos sociales actuales en un proceso de, al menos, treinta años, relacionando este ciclo de movilizaciones con el ciclo de protestas que dio origen al proceso de transición política, lo que Garcés denomina el "primer movimiento social antineoliberal de América Latina" (p.23), el que fue desactivado -el aletargamiento del que habla Grez- a partir de la constitución de una "transición enajenada", "expropiada a los movimientos populares chilenos" (Ibid.).

Otra dimensión a analizar se plantea en el capítulo titulado “¿Qué es un movimiento social?", que resume los derroteros de este concepto en la teoría social. Allí, Garcés pasa revista a la lectura movimiento social $=$ movimiento 
obrero que predominó hasta los años 70, la que sólo fue cuestionada, primero -y parcialmente-, por la Teoría de la Marginalidad de los 60 y luego, en los 80, por la inclusiva categoría de movimiento popular, planteada "como la suma o la confluencia de diversos movimientos de origen popular y que compartían un ideario de transformación socialista de las sociedades latinoamericanas” (p.31).

Lo interesante es que, luego de este pequeño recorrido continental, Garcés se remite exclusivamente al estado del arte de los debates norteamericano y europeo, en los que la propuesta de Sidney Tarrow (1997) y Alberto Melucci (1994) serían su síntesis, respectivamente. Tarrow profundizando en el cómo de la acción colectiva, poniendo el acento en la noción de repertorios de acción, de confrontación con los oponentes y de oportunidades políticas; y el italiano, profundizando en el porqué interno del movimiento, enfatizando en la construcción identitaria del sujeto colectivo (Garcés, 2012, pp.33-39). Ahora, cabe preguntarse: ¿hasta qué punto este análisis es aplicable al caso chileno? Pregunta clave cuando se considera, por ejemplo, la escasa relación que se da entre la sociedad civil y la política institucionalizada -elemento clave para la primera entrada de análisis-, la que se refleja en la débil relación entre los movimientos y los partidos políticos. Garcés no evade el tema y advierte la necesidad de que todo estudio que busque entender la realidad latinoamericana, desde la perspectiva de los movimientos sociales, debe partir del reconocimiento de:

(...) las formas de relación que se han establecido entre los Estados y los grupos y clases populares, la mayor parte de las veces con fuertes componentes represivos como producto de una defectuosa construcción de Estado, en el sentido de la democracia y de la integración social, o de formas de integración parcial y segmentada, que incluye a algunos y excluye a otros. Por otra parte, se trataría también de reconocer y valorar las diversas iniciativas de asociación y movilización de los grupos sociales populares, de las capacidades y 
recursos que se pusieron en movimiento, de las memorias de esas luchas y de las identidades que en esos proyectos emergieron o se recrearon. Si se tienen en cuenta ambas perspectivas, lo que resulta crecientemente una certidumbre para quienes investigan y piensan la historia social de América latina, es que una de las principales "formas de hacer política" de nuestros pueblos es a través de la constitución de movimientos sociales. (p.46)

Visto así, un análisis de "las resistencias y de las alternativas de cambio" no puede limitarse sólo a una mirada desde arriba, enfocándose en el enfrentamiento con los oponentes, en los espacios de la política institucional y sus alrededores -la calle-, sino que debe incluir una mirada desde abajo, que enfoque y amplíe aquellos espacios donde se configuran esas alternativas. Dicho giro o cambio de lente es recogido parcialmente por Garcés cuando señala:

La constitución de un movimiento social supone necesariamente el desarrollo de alguna forma de organización y redes de organizaciones sociales, así como de recursos culturales e identitarios que se irán modificando según se desenvuelve la propia acción colectiva (...) En su mayor desarrollo, los movimientos sociales pueden encarnar proyectos de cambio social que afecten parcial o globalmente el sistema de dominación. (p.45)

¿Cuánto hemos avanzado en el análisis para distinguir con claridad, en el mediano plazo esos espacios donde se está encarnando dicho proyecto de cambio social? 


\section{GABRIEL SALAZAR Y EL 'MOVIMIENTO SOCIAL-CIUDADANO'}

Salazar se ha transformado en uno de los intelectuales más reconocidos del último tiempo en Chile, considerado por los mass media como uno de los intelectuales detrás del movimiento estudiantil del 2011. Sus intervenciones académicas y no- sobre esta temática son múltiples pero derivaron en un extenso trabajo titulado Movimientos Sociales en Chile. Trayectoria histórica y proyección política (2012) en el cual ofrece una lectura de largo aliento (dos siglos) de evolución de los movimientos sociales. En esta obra, Salazar realizó una traducción de sus tesis sobre la sociedad popular chilena integrando la categoría de movimiento social, categoría que parece (re)surgir como consenso discursivo global para nombrar el proceso de aparición del "sujeto" (desde fines de los 60), acrecentado hoy frente al colapso de los Mega-Estados (hacia 1982) y de las crisis sucesivas (1997, 2008-2009) de los Mega-Mercados. Así, bajo esta nueva categoría de análisis Salazar vuelve a repasar nuestra historia republicana analizando, por un lado, el avance de la "anomia anticívica" -como la denomina- del sistema constitucional chileno (desde 1823) y, por otro lado, las respuestas ciudadanas (autonomía popular, acción sociocrática, peticionismo-movimiento de masas y movimiento ciudadano).

Desde este enfoque, el ciclo de movilización no podría ser entendido sin centrarse en los procesos de mediana duración que lo contienen $\mathrm{y}$, en particular, al período que se abre desde 1947 y que llegaría hasta nuestros días el "quinto cambio de conducta ciudadana" (p.43)- en la que la ciudadanía habría reaccionado, primero, contra el conservadurismo constitucional y la esterilidad gubernamental de la clase política populista (en la fase 1947-1973), para retomar esta reacción en el período 1976-1990 frente al orden neoliberaldictatorial impuesto. Estos dos procesos son leídos, por el autor, como la gestación de una soberanía ciudadana-popular que, adentrándonos en nuestra historia reciente, habría sufrido una "doble traición continuista" (por parte de 
las clases políticas -civil y militar-): primero, a partir del retorno hegemónico de la vieja clase política civil y, segundo, a través de la consolidación conservadora del modelo neoliberal impuesto ilegítimamente por el terrorismo militar" (Ibid.). Doble traición que explicaría la también doble crisis de representatividad y legitimidad presente y la reacción ciudadana, luego de quince años (1990-2006), a partir de la denominada "Revolución Pingüina".

Acá, aunque hay una lectura que analiza los porqués externos del fenómeno -en concordancia general con los autores anteriores-, la apuesta salazariana va por el lado de dilucidar, en el mediano y largo plazo, los porqués internos del "movimiento social-ciudadano". Lectura donde la memoria histórica, entendida como fuente de sentidos, ha tenido el rol fundamental de entregar los códigos con los cuales los sujetos han leído y procesado su condicionamiento externo. Bajo esa línea argumentativa, en el Chile actual estaríamos en presencia de una memoria "sobrecargada" al ser depositaria de “tres memorias vivas simultáneas de 'soberanía incipiente': a) la del poder popular de fines de los sesenta, b) la de la supervivencia comunitaria de los ochenta, y c) la de la lucha por todos los medios contra la dictadura, también de los ochenta" (Ibíd.). Así, el "estallido pingüino" -y, por añadidura, las movilizaciones del 2011- eran cosa de tiempo, en tanto "si esas tres formas de soberanía formaban parte viva de su memoria, las tres sumaban un poder de acción instalado que podía desplegarse en cualquier momento y orden, según coyuntura, por opción, o por rutina" (Ídem.).

Para Salazar, la transición concertacionista ocultó bajo un discurso triunfalista el "proceso profundo" de toma de conciencia ciudadano que se gestaba mirando las "anomalías congénitas" que generaba el modelo neoliberal a nivel comunitario con la mercantilización de funciones centrales de la vida social: educación, salud, cultura, empleo, etc. (p.45). Proceso profundo de "propensión autonómica" que, sobre el soporte de una cultura popular, expresada en el "cabildeo cultural ciudadano" de las asambleas territoriales y la 
práctica de la autoeducación popular, adquiere "correlatos de propuesta alternativa" como bases de un proyecto "constitucional alternativo". Es decir, algo mucho más profunda que el "inventario de 'NO's" que se expresa en el espacio público (Ibíd.).

A la hora del balance, la propuesta salazariana abre dos frentes de análisis: primero, el temporal, donde a partir de una mirada mediano y largoplacista, saca el fenómeno de la coyuntura y lo coloca en sintonía con una serie de procesos específicos de los últimos 65 años (1947-2011) lo que amplía la mirada analítica desde lo 'juvenil' hacia otros sectores sociales en movimiento. Segundo, el de los porqués internos, otorgando mayores claves interpretativas para analizar la forma de procesar el condicionamiento de los factores externos (la construcción de una cultura popular), aunque sin profundizar en sus cómo internos, los que quedan anunciado cual titulares (las prácticas de autoeducación y de asambleísmo).

Pero Salazar reserva hacia el final una revisión de la "Teoría general de los movimientos sociales" que es clave para develar y entender sus presupuestos teóricos. Similar a Garcés, la bibliografía revisada por Salazar es extensa e incluye una reseña del debate clásico (pos 68) y un interesante resumen del debate anglosajón entre 1991 y 2002. Su condena es certera: la lectura propuesta, casi en su totalidad desde la sociología, “es menos 'sistémica' de lo que parece, pues reproduce el paso-a-paso del cambio señalado, el contexto cultural del lugar donde ha ocurrido, como también la específica historicidad de la 'teoría"' (406). Así, el debate europeo habría pasado del análisis de la "conducta colectiva" (el cómo externo, parafraseando a Pérez Ledesma) propio del estructuralismo-funcionalista, a la "movilización de recursos" (un cómo interno), hasta llegar al concepto de "cultura social", que correspondería a un porqué interno desde el cual se proyectan otros análisis complementarios. Sin embargo, plantea Salazar, esto habría decantado en el concepto de "cultura social", en tanto el "poder" del movimiento estaría en el 
"cuerpo cultural" que es capaz de conformar y proyectar. Es desde esta afirmación que el autor construye su definición de movimiento social:

La razón histórica que motoriza los movimientos sociales es, pues, una 'cultura' propia, que, en diversos grados, no es ni puede ser la misma del sistema dominante, puesto que la mayoría de los MS intenta ajustar por sí mismo, con sus propios diseños y herramientas, los desperfectos sectoriales de ese sistema, o cambiarlo por completo. Por tanto, el movimiento se inicia cuando los sujetos sociales perciben o sufren los dichos 'desperfectos', sigue cuando ellos van desarrollando sus recursos culturales estratégicos, y madura cuando, en un momento dado («oportunidad política») el movimiento emerge en el espacio público para realizar los cambios (parciales o totales) que señalan sus objetivos. Todo movimiento social implica, pues, una vida histórica temporalmente acotada (pasajera), y en él, como quiera que sea el resultado final de su intervención en el ámbito político, la sustancia real de su poder socio-político radica en la calidad y potencialidad histórica de la cultura propia que ha logrado consolidar. Y esto implica un proceso de aprendizaje colectivo. (pp. 414-415)

La síntesis es sugerente porque deja en el centro del análisis esta "cultura propia" desde la cual se articulan los diferentes cómo que este sujeto colectivo desarrolla (hacia dentro -su propia formación y proyección- y hacia fuera -la lucha con sus oponentes-); y porque, como decíamos anteriormente, en dicha "cultura" se sostendría el poder socio-político del sujeto colectivo (y, con ello, su proyecto). Sin embargo, Salazar busca llegar más lejos, planteando que si analizamos las prácticas autoeducativas de los movimientos (entendidas como el proceso colectivo y comunitario de sistematización y aprendizaje de microexperiencias de resistencia y liberación, que se "archivarían" en la memoria social del movimiento), éstas nos denotan que los cambios venideros ya existirían en potencia o a nivel micro en la vivencia del mismo movimiento, 
reservándose éste la posibilidad de introducirlos -o no- en la sociedad global. Este giro cambia de forma radical nuestro foco de atención. ¿Qué sería lo que vemos efectivamente en escena pública cuando se mueve un movimiento? ¿Sería sólo una irrupción, distorsionada por el enfrentamiento con los oponentes, de un proceso oculto pero de mayor profundidad? Porque, de ser así -como plantea el autor-, la preocupación por la proyección de los movimientos en la "política realmente existente", como planteaba Grez, sería una preocupación secundaria, y deseable sólo si esto no coloca en peligro el proceso de (auto)construcción cultural que subyace al movimiento, el que no se gesta en la "breve vida pública" del movimiento sino, precisamente en su latencia.

En este punto del debate en que Salazar ya (re)configuró una propuesta analítica de cara a las reflexiones primermundistas, el autor abre el debate hacia Latinoamérica. Sin embargo, acá su análisis tiende a desactualizarse; si bien hace una consistente lectura del debate hasta comienzos de los 90, desde esa fecha en adelante se queda en los titulares -con escasa profundización-, reduciendo la lectura a la crisis del Estado -evidente desde el fin de las Dictaduras-, el avance del neoliberalismo y su cuestionamiento "desde abajo", que habría generado una tendencia a leer a los movimientos "desde sí mismos" (pp. 428-435).

\section{UN PRIMER BALANCE DE LAS LECTURAS DE EMERGENCIA DE LA NHS}

¿Qué podemos aportar como corriente disciplinaria al debate general sobre movimientos en Chile? Partamos por algunos consensos: la NHS, en base a tres de sus representantes, tiende a coincidir en líneas gruesas entre sí y con el debate nacional reseñado al comienzo de este trabajo, en el diagnóstico de las causas sistémicas -o los porqués externos- que explican el resurgimiento de los movimientos sociales en el Chile actual: el corsé institucional heredado de la 
Dictadura, su escasa porosidad para procesar demandas sociales y la doble crisis de representatividad/legitimidad -en el plano de lo político-estatal-, sumado al avance arrollador del modelo neoliberal -en el plano económico-. Igualmente, existiría un segundo consenso disciplinar en relación a la categoría adecuada para definir los procesos de asociatividad y organización de este sujeto colectivo en movimiento (movimientos sociales).

Pero, por otro lado, existen disensos con este debate nacional que giran en torno a los roles que se le asignan a este sujeto colectivo y su potencialidad. Así, si el primer balance de las ciencias sociales locales daba cuenta de una mirada que tendía a centrarse más en los modelos que en los sujetos -donde estos últimos tienden a ser vistos como fuerza a conducir más que como colectividades deliberantes o constitutivos/constituyentes de proyecto-, el análisis de la NHS cuestiona esta mirada al proponer entradas a partir de los sujetos, equiparando la necesidad de estudiar tanto la vida de los modelos (político y económico) como la acción colectiva de los sujetos, superando parcialmente la visión que asocia el movimiento social con el ejercicio de la "negatividad" (identificación de problemas, peticionismo, reducción a demandas parciales), devolviéndole historicidad (o "positividad") al defender la idea de la creación de proyecto desde fuera de la política estatal.

Sin embargo, este giro hacia el sujeto no ha generado consensos al interior de la corriente disciplinar, lo que se evidencia, por ejemplo, al comparar las vías de salida al "empate catastrófico" -como señalara Garcés- y las estrategias de resistencia a las "anomalías congénitas" del neoliberalismo. Para el análisis de Grez, por ejemplo, la clave para el éxito de los movimientos está en la dimensión externa. En tanto los movimientos se ubican en el seno de la sociedad civil -y precisamente por estar en dicha posición-, deben apuntar sus acciones colectivas hacia la incidencia, ojalá directa, en la sociedad política. Garcés recoge esta lectura pero no limita el análisis a la relación exclusiva del movimiento social con la sociedad política. Asumiendo el carácter histórico del 
Estado en Chile (incompleto, refractario al cambio), y la tradición de nuestra historia política (colmada de pequeñas historias de resistencia y alternativa emanadas desde -y para- la sociedad civil), busca devolver la atención a la acción colectiva de los movimientos, destacando el rol que ésta tiene tanto para articular públicamente el malestar, como para generar colectivamente proyectos emancipadores que puedan, eventualmente, llevar al proyecto de dominación a una crisis general que obligue a reformas más o menos profundas (única posibilidad de reforma en las actuales condiciones) o, en su grado máximo, que sustituya un orden por otro. Así visto, los movimientos pueden seguir siendo considerados como una fuerza, pero con autonomía para resistir y decidir e historicidad para proyectarse.

Pero si Garcés abre la posibilidad a un análisis interno de los movimientos, Salazar lleva esta idea hacia el status de única lectura posible, ya sea en la fase de visibilidad (breve) o de latencia (profunda) de los movimientos, destacando esta última fase en tanto en ella se fraguaría no sólo la crítica al modelo de dominación sino también la prefiguración de las alternativas a éste.

Con todo, la lectura de la NHS no desbanca el análisis de la dimensión externa (estatal) de los movimientos, apostando a complementar este análisis con uno que, al privilegiar al sujeto, se vuelve cada vez más interno y largoplacista. Sin embargo, esto ha derivado en visiones que conciben esta opción epistemológica, en un grado de aplicación mínimo, como un complemento al análisis estructural o, en su grado máximo, como la puerta de entrada a una dimensión más profunda y proyectiva de los sujetos. Es precisamente la apertura a esta nueva dimensión de análisis -sumada a la variable temporal- lo que podría explicar cierto "triunfalismo historiográfico" que se nos atribuye, a pesar de las diferencias ya vistas. Finalmente, aunque el debate no está terminado en este punto, es preciso reconocer y destacar que el camino que llevó de la revalorización de la acción colectiva al reconocimiento 
de la capacidad proyectiva del sujeto, y de la visibilización de la dimensión interna (sus porqués y sus cómo) a la valoración de la latencia (como espacio fundamental de análisis), se constituye como el aporte más consistente de la NHS al debate en curso del resto del pensamiento crítico nacional.

Antes de cerrar este apartado, una pregunta se asoma. Si bien existe consenso en la categorización del sujeto colectivo (movimientos sociales), ¿hasta qué punto ese consenso es parte de una reflexión en torno al tipo histórico de sujeto que se articula en esta región? ¿Corresponderá más bien a un acuerdo operativo, una adopción temporal de algunas categorías europeas para leer con celeridad nuestra realidad latinoamericana y así levantar hipótesis que permitan estudiar la aparente novedad del fenómeno? Difícil saberlo. Lo que sí sabemos es que dichas preguntas nos llevan, irremediablemente, a otra en la que si podemos aventurar respuesta: ¿de qué forma produce su teoría la Historia Social? O, más específico aún, ¿con quiénes está dialogando en su reflexión?

Una respuesta rápida -e ideal- sería plantear que la teoría de la NHS en Chile viene del diálogo con los sujetos en movimiento, pero esta reactivación del diálogo disciplina-sociedad es reciente, gatillada por las necesidades autoeducativas del mismo movimiento. La tónica de los últimos años ha sido, de hecho, la distancia academia-sociedad, la que pasó de ser resentida por las organizaciones sociales y juveniles, a ser superada por éstos al producir su propia teoría sin necesidad de la academia (Artaza, Fauré y Poch, 2013, pp. 7695). Pero el problema no termina allí ya que, como hemos visto, las referencias a la teoría siguen estando cargadas a los debates primermundistas, con un franco desconocimiento de las reflexiones que se realizan al sur del Río Grande. Problema en tanto el escenario continental ya acumula tres décadas de presencia sistemática e ininterrumpida de movimientos sociales, tanto en sus escenarios públicos como en sus trastiendas, generando una serie de transformaciones en nuestras sociedades que han estado acompañadas de un nutrido -aunque, a ratos, desordenado- debate que claramente podría enriquecer 
nuestra incipiente lectura nacional. Ahora, ¿qué grados de sintonía tiene la reflexión de las ciencias sociales en Chile con las desarrolladas por el pensamiento crítico latinoamericano? ¿Qué puentes de (retro)alimentación se pueden establecer? Hagamos un breve recorrido.

\section{EL DEBATE LATINOAMERICANO RECIENTE: DE LAAUTOPRODUCCIÓN SOCIETAL AL AUTONOMISMO}

La implantación del neoliberalismo en América Latina, en paralelo o posterior a la oleada de dictaduras que asolara el continente desde 1964, implicó una reestructuración de los Estados, los mercados y la sociedad civil. El retroceso de los primeros frente al avance de los segundos desestructuró a los actores fundamentales del tercero, comenzando un proceso de reconfiguración social que hizo visible, desde los márgenes, a los sin: sin tierra, sin casa, sin trabajo, sin derechos. Sujetos que se resistían a un análisis estructural estático en tanto podían, por ejemplo, ser leídos en forma simultánea como movimientos clasistas pero con directrices étnicas o de género o movimientos culturalistas con orientaciones clasistas, con mutaciones constantes a partir de ese mover-se continuo.

Es por ello que el debate que buscó comprender este fenómeno fue -y sigue siendo- un desafío permanente y siempre inconcluso y, en tanto es un relato extenso, nos centraremos sólo en tres momentos -de los últimos 25 añosen los que, creemos, se han dado reflexiones que permitirían problematizar y enriquecer el debate en Chile. 


\section{FINES DE LOS 80: ¿Y SI SIEMPRE FUE ASÍ Y NOSOTROS NO LO VIMOS?}

Los primeros intentos sistemáticos de comprensión de la nueva realidad social latinoamericana -a ras de sociedad civil- se dieron con fuerza a fines de la década de los 80. El fin de las Dictaduras y el debate en torno a qué modelo de pos dictadura se podía seguir, llevó a las ciencias sociales a analizar el guion que le correspondería a estos movimientos otros en el nuevo marco democrático. Así, estas ciencias sociales procesaron esta nueva realidad -y a sus movimientos- enfrentando un dilema no menor: ¿con qué categorías leer lo nuevo? Esto las llevó, en general, a seguir dos caminos: en un extremo, procesar la novedad integrándola a los marcos interpretativos existentes (leer lo nuevo con categorías antiguas) o, en su extremo opuesto, desechar las categorías buscando captar la esencia de aquello que se anuncia.

Más cercanos a la segunda opción (aunque minoritaria en la época), el boliviano Fernando Calderón y su par argentina Elizabeth Jelin se empecinaban, hacia 1987, en esta empresa de comprender lo nuevo. Cualquier análisis, planteaban, debía partir de una condicionante histórica: que en América Latina el Estado -que había obnubilado el análisis de las ciencias sociales- fue, en muchos sentidos, no sólo un "producto" de la sociedad, sino también "productor" de ella, lo que dejaba al continente como "(...) una región donde los procesos de lucha, la conflictualidad asociada a los procesos de transformación se manifestaron mucho más como conflictos dentro del Estado que como luchas estrictamente sociales" (Calderón y Jelin, 1987, pp. 173-174). Sin embargo, esa condición histórica no podía cegar el estudio o negar las características disímiles que presentaba el fenómeno nuevo que aparecía: la constitución simultánea en el continente de "movimientos sociales" que si bien podían enfocarse en la constitución de "formas orgánicas de acción social por el control del sistema político y cultural”; planteaban también -a veces en forma simultánea- "modos de transformación y participación cotidiana de 
autoproducción societal” (p.176). Calderón y Jelin, claramente, habían visto más allá del Estado. Y este esfuerzo los aventuraría a descubrir que la acción colectiva no sólo buscaba un impacto fuera de sí misma, sino fundamentalmente dentro de sí. La conclusión era radical: los movimientos, en su acción, no sólo constituían una política otra sino, más allá aún, se sentaban las bases para la constitución de una sociedad otra (p.177).

El giro de Calderón y Jelin, centrado en rescatar y poner en valor la acción colectiva de los movimientos más allá de la omnipresencia estatal, generó dos cosas clave (que serían retomadas en los debates posteriores en el continente): en primer lugar, devolvió la historicidad al sujeto colectivo, monopolizada por la élite o concentrada en sus instituciones; y, en segundo lugar, al seguir la pista a la acción colectiva, iluminó las dos dimensiones en que esta se materializa, la externa y la interna, marcando una pauta para las investigaciones posteriores, sobre todo en relación a la segunda opción (Cf. Calderón, 1995). Conscientes de lo original de su análisis, los mismos autores no dejaban de sorprenderse ante el (re)descubrimiento de este caudal colorido de historicidad detrás de los blancos muros del Estado: "La pregunta que surge de inmediato, imposible de responder a ciencia cierta, es si se trata de una 'nueva realidad' o si la vida social siempre fue así y nosotros, ciegos por el peso de los dogmas dominantes, no la estábamos viendo" (Calderón y Jelin, 1987, p.177).

\section{ZIBECHI Y LAS 'TENDENCIAS' (DESPUNTANDO EL SIGLO XXI)}

Quince años después de la reflexión de Calderón y Jelin, mucha agua y muchos movimientos habían pasado bajo el puente latinoamericano. Algunos que recién se constituían o aparecían en escena en esos años se habían transformado en referentes obligados (Ejército Zapatista de Liberación 
Nacional, Movimiento de Trabajadores Rurales Sin Tierra, movimientos indigenistas en Bolivia y Ecuador, movimientos piqueteros en Argentina), y en su remezón telúrico, habían logrado cuestionar el modelo neoliberal a partir de una serie de prácticas de resistencia que, aunque no necesariamente atacaban de forma directa al Mercado o al Estado, terminaron reconfigurándolos parcialmente.

En el año 2003, Raúl Zibechi, periodista y educador popular, intentó hacer un nuevo balance. A diferencia de los autores anteriores, Zibechi se montaba sobre una ola movimentista que, desbordada, ya dejaba consecuencias en las tierras firmes de la política estatal y la economía neoliberal: "De esta forma los movimientos forzaron a las elites a negociar y a tener en cuenta sus demandas, y contribuyeron a instalar gobiernos progresistas en Venezuela, Brasil y Ecuador. El neoliberalismo se estrelló contra la oleada de movilizaciones sociales que abrió grietas más o menos profundas en el modelo" (Zibechi, 2008, p.13).

Pero esa ola no fue presenciada por Zibechi como espectador académico, sino como un investigador militante. Por ello, rechazó la idea de plantear características estáticas o definitivas y se enfocó en mostrar las "tendencias" de ese "estar siendo" colectivo que denominó "nuevos movimientos sociales": en primer lugar, la territorialización, una respuesta estratégica a la desterritorialización productiva llevada a cabo por las dictaduras y el neoliberalismo para eliminar aquellos espacios donde los viejos movimientos habían ganado poder y sentido; segundo, la búsqueda de autonomía tanto material como simbólica; tercero, la revalorización de la cultura y la formación de la identidad; cuarto, la preocupación creciente por su propia formación y la formación de sus propios intelectuales; quinto, el rol protagónico de las mujeres en los movimientos; sexto, una preocupación por la organización justa del trabajo y una relación armónica con el medio ambiente; séptimo, el cambio en las "formas" o "repertorios de acción", de aquellos de 
carácter instrumental a otros más "autoafirmativos", donde destaca la "toma", como ocupación que hace visible y reafirma la identidad"(Zibechi, 2008, pp. 13-17).

Siguiendo la línea argumentativa de Calderón y Jelin, la lista de "tendencias" que develó Zibechi no sólo giraba en torno a la historicidad -que empezará a conceptualizarse derechamente como poder (Colectivo Situaciones, 2001; Holloway, 2002)- sino que, además, terminaba con la mirada fija en la dimensión interna del fenómeno, escenario de la territorialización. Para el uruguayo, sería sobre este espacio comunitario y recuperado donde los movimientos leen sus condicionamientos y se re-conocen como sujeto colectivo a partir de una serie de prácticas donde enfrentan colectivamente la vivencia material, concreta, cotidiana- del modelo neoliberal.

\section{DEL AUTONOMISMO Y LA CONFUSIÓN EN TORNO AL PROGRESISMO (LA ACTUAL COYUNTURA)}

Diez años después de las provocaciones de Zibechi, ¿qué ha cambiado y que ha permanecido en el debate latinoamericano? Partiremos señalando que este debate ha tenido, en estos últimos años, una revitalización, diversificación y multiplicación importante en el continente. El fenómeno de los movimientos sociales no se ha detenido y, por tanto, tampoco lo ha hecho la reflexión académica o no- sobre este tema. Es más, el escenario continental que soporta a estos movimientos entró en transformaciones significativas y novedosas, complejizando la posibilidad de reflexión, en tanto la oleada de movimientos sociales de los 90 ayudó a deslegitimar el modelo neoliberal abriendo el paso a los gobiernos progresistas e izquierdistas de nuevo tipo en importantes sectores del continente (y que parecen estar cerrando su ciclo al momento de escribir estas líneas). Un avance democratizador que no remeció el modelo económico, 
sino que eliminó sus excesos (privatizaciones) manteniendo la lógica extractivista (o de "acumulación por desposesión”) intacta (Zibechi, 2011). Así, como señala el uruguayo:

El cambio más importante registrado en la región es la convivencia entre un poderoso y autónomo capital transnacional y un relativo fortalecimiento de los Estados de la mano de gobiernos progresistas. Ambos hechos son, evidentemente, contradictorios, pero esbozan un campo de fuerzas de notable dinamismo en el cual la tendencia es a la confrontación entre movimientos y extractivismo, lo que coloca a los Estados en una situación paradójica: necesitan del modelo para lubricar los ingresos fiscales que den sustento a sus políticas, pero también necesitan el apoyo de los movimientos sin los cuales su legitimidad queda en cuestión. (p.21)

¿Qué ha hecho el pensamiento crítico frente a este nuevo escenario?, ¿por dónde han ido sus reflexiones? El Observatorio Social de América Latina decidió hacer un recuento el 2011 de lo debatido en los últimos 10 años. Entre las características que señalaban los autores convocados por OSAL sobre la reflexión crítica en el continente, se pueden señalar:

1) la intelectualidad se ha fraccionado y diversificado: mientras algunos críticos de ayer hoy se han "gubernamentalizado", limitando su reflexión al apoyo o justificación de las políticas del progresismo, siempre tomando como referencia central a los gobiernos, el campo crítico ha recibido, como refresco, el ingreso de nuevas generaciones de jóvenes, no provenientes del mundo académico. "Pensadores/activistas" o "investigadores/militantes" que han devuelto las miradas a la cotidianeidad de los movimientos y a temáticas no trabajadas anteriormente (Iglesias, 2011, pp. 22-23).

2) Se ha generado una revisión crítica de las categorías conceptuales utilizadas, buscando despojarse de la condición de "colonialidad del saber" que imperaría en el pensamiento crítico de la región Esto se ha hecho patente al 
constatar, por ejemplo, que la noción central de "territorio" no es considerada por las lecturas primermundistas, lo que implica que la adopción acrítica de estos conceptos dejaría fuera un elemento central en la constitución de "nuestros" movimientos (Zibechi, 2011a, p. 68).

3) Lo anterior ha significado una "explosión discursiva" o "turbulencia de conceptos", aunque persista la hegemonía de la categoría movimiento social (Iglesias, 2011, p. 28).

Ahora, ¿qué "claridades” -como les llama Mónica Iglesias- ha traído la reflexión? Las más importantes, a nuestro parecer, son:

1) Se han establecido dos grandes corrientes: el hegemonismo y el autonomismo, que tendrán, como sus voceros principales, a Emir Sader y Raúl Zibechi, respectivamente. Así, aunque exista consenso en la centralidad que adquiere en esta etapa la necesidad de que los movimientos construyan alternativas al modelo político y económico, el cómo ha generado discrepancias, por ahora, irreconciliables (sobre todo en torno a la revitalización del rol de los partidos y del Estado en este proceso, relacionado con el auge de los gobiernos "progresistas").

2) Se han incorporado nuevos temas. Entre estos, ha aparecido con fuerza la reflexión en torno a la "proyección prefigurativa de la nueva sociedad, o sea la multitud de emprendimientos productivos, educativos y de salud que (los movimientos) han puesto de pie mientras resisten al modelo" (Zibechi, 2011, p. 22).

3) Por ello, la mirada hacia el interior de los movimientos ha cuestionado su definición en base al "moverse" en el espacio público, reinstalando la necesidad de mirar los procesos de latencia como otra dimensión del mismo movimiento (lo que se ha acrecentado a partir de la constatación del "reflujo" movimentista en el espacio público -o dimensión externa- en directa proporcionalidad, por ejemplo, al avance del progresismo político). 
4) Esa misma mirada interna ha remarcado la constatación de la heterogeneidad de los movimientos, lo que complejiza la comprensión y proyección de éstos. Por lo mismo, se ha instalado la necesidad de "recuperar la historicidad de los movimientos sociales (ya que), su sociogénesis, es fundamental para dilucidar los procesos de constitución de subjetividad(es) política(s)" (Iglesias, 2011, p. 31).

\section{BALANCE FINAL Y PREGUNTAS FUTURAS PARA UNA HISTORIA SOCIAL DEL MOVIMIENTO ESTUDIANTIL}

Con todo, a la hora del balance, varias cosas salen a la luz. En primer lugar, que la pregunta de Calderón y Jelin (si lo que veían constituía una "nueva realidad" o siempre existió pero las ciencias sociales no fueron capaces de verla), un cuarto de siglo después aún no se responde del todo. Sin embargo, algunas cosas si se han afirmado en este tiempo. Por ejemplo, lo anunciado por ambos autores con timidez en los 80 , parece confirmado por la investigación continental: los movimientos en su andar auto producen sociedad. Tanto fue confirmado esto que fue necesario hacer un derrotero que hiciera visible esta nueva sociedad en eterno parto: se le cartografió (la "territorialización" de Zibechi), caracterizando luego las pequeñas y grandes acciones que -sin modelo único, sin Estado, sin teoría social- constituyeron un acervo de saberes que el mismo movimiento socializó horizontalmente, constituyendo así su "proyección prefigurativa" (Zibechi), otra forma de decir proyecto histórico, como una sistematización permanente de sus luchas cotidianas.

Ese camino impactó al pensamiento crítico latinoamericano que, emulando el proceso, tendió a cuestionar sus propios preceptos teóricos, abandonando categorías e inventando otras, encarando el desafío que les hicieran Calderón y Jelin el 87 de "curar la ceguera de los dogmas dominantes”, 
aunque sin síntesis hasta hoy (por lo que decíamos que la pregunta de los autores no se contesta del todo). Sin embargo, pareciera que -tomando precauciones-, el pensamiento crítico latinoamericano está decidiendo ampliar la mirada, no sólo a lo visible en el espacio público, sino también a la profundidad de la latencia, invitando a una mirada cada vez más histórica (y no coyuntural) de los procesos. Así, la ardua empresa de construir categorías que nos permitan aprehender la realidad sigue palpitando con urgencia (y, en esa empresa, el concepto mismo de movimiento social ha resultado herido, pero no muerto). Es por ello que, asumiendo la complejidad y renunciando en parte a la universalidad, la necesidad de definir tipos históricos de movimientos sociales que instala el presente nos invita, de nuevo, como NHS y como sujetos comprometidos con el cambio social, a trabajar.

Pero lo anterior ha dejado en evidencia también un desafío político no menor. El énfasis dado a la dimensión interna -donde, en mayor medida, se fraguaría el proyecto- hizo restar importancia al avance o retroceso en la dimensión externa, casi como un voto de confianza a la labor transformadora que pudieran realizar los gobiernos progresistas. Hoy, cuando se verifica que estos gobiernos de nuevo tipo han hipotecado el avance en protección social con una sobrevida al modelo neoliberal, y que comienzan su retirada por el arribo de una nueva corriente derechista conservadora, la confusión por parte de los movimientos se tradujo en confusión en las ciencias sociales. Pero este impasse, que puede y debe resolverse generando teoría que explique el nuevo contexto (extractivismo con gobiernos progresistas y reconfiguración de la derecha), no debe dejar de lado la dimensión donde se construyen los proyectos alternativos, hoy más necesarios que nunca. Eso parece ser lo que anuncia la reflexión última en el continente, y concordamos plenamente con la tarea.

Ahora, ¿qué implica hacer dialogar este acumulado latinoamericano con la reflexión local y, en específico, de la NHS al momento de trabajar una historia social del ciclo de movilización estudiantil pasado y en curso? Sabemos 
que debe darse un necesario balance investigativo entre las dimensiones externa e interna pero, en un escenario de neoliberalismo sostenido y maduro como el nuestro, ¿no debiera darse mayor énfasis en el cómo y porqué de la dimensión donde puede configurarse la propuesta alternativa?

Sin embargo, ello implicaría incluir tres cuestiones básicas que recogemos de la reflexión continental y que no se responden fácilmente para el caso estudiantil:

1) Desde una mirada general, urge resolver la pregunta por la "autoproducción societal" del movimiento estudiantil. ¿Qué propuesta ha configurado en este último ciclo de movilización? ¿Es de carácter generacional o transversal, enfocado en lo educativo o a nivel societal general?

2) En lo que refiere a la territorialización: ¿cuál es el territorio donde se encarna con mayor fertilidad esta autoproducción societal? ¿En las escuelas, liceos o universidades ocupados a la fuerza en las movilizaciones, en sus territorios locales-poblacionales, o en los espacios céntricos ocupados en cada marcha? ¿Qué implica cada uno de estos niveles?

3) La tercera cuestión es la proyección prefigurativa: ¿cómo se operativiza esa sistematización de las experiencias prefigurativas que permiten su proyección? ¿Se da de manera implícita o explícita? ¿Se gestiona autónomamente, es realizado por intelectuales de base, con apoyo de ONG, o en modelos mixtos? ¿Qué caminos metodológicos se siguen?

Todas estas preguntas pueden y deben ser contestadas con urgencia. Para ello, la NHS en específico -y las ciencias sociales en general- requieren integrarse al debate latinoamericano para avanzar en reflexiones decoloniales que puedan constituirse en insumos situados y fechados para los sujetos en movimiento. Solo desde la proliferación de investigaciones territorializadas, a medio camino entre la sistematización de experiencias y la investigación académica en su formato clásico, es que se constituirá un archivo desde el cual, 
en una segunda fase, surgirán las categorías necesarias y acordes para la lectura de la realidad latinoamericana pasada y presente.

\section{REFERENCIAS}

Álvarez, A. (2008). "Tenemos razón y somos mayoría": Prácticas asociativas en el Movimiento Estudiantil secundario Chileno (2000-2008). (Tesis de grado). Universidad de Chile: Santiago.

Artaza, P., Fauré, D. y Poch, P. (Ed.). (2013). Diálogos sobre memoria social, sistematización de experiencias y organización juvenil popular. Santiago, Chile: Facultad de Filosofía y Humanidades, Universidad de Chile.

Atria, F. (2012). La mala educación. Ideas que inspiran al movimiento estudiantil en Chile. Santiago, Chile: Catalonia-Ciper. .

Calderón, F. y Jelin, E. (1987). Clases sociales y movimientos sociales en América Latina. Perspectivas y realidades. Revista Proposiciones 14, pp. 173-189.

Colectivo Diatriba, OPECH, Centro Alerta (2011). Trazas de Utopía. La experiencia de autogestión de cuatro liceos chilenos durante 2011. Santiago, Chile: Quimantú.

Durán, C. (2011). El acontecimiento estudiantil y el viraje del proceso sociopolítico chileno. Revista OSAL Año XIII, N 31, pp. 39-60.

Fauré, D. (2011). De estudiantes, cacerolazos y memoria histórica. Recuperado de http://www.elciudadano.cl/2011/09/07/40537/de-estudiantescacerolazos-y-memoria-historica/

Fauré, D. (2012). Cultura, rebeldía y educación popular: Reflexiones en torno a la historicidad de los 'nuevos movimientos juveniles' (Chile, 19992008). En D. Fauré y L. González. Somos andando. Prácticas, caminos y saberes para construir Educación Popular hoy (pp. 19-35). Santiago, Chile: Quimantú.

Garcés, M. (2012). El despertar de la sociedad. Los movimientos sociales en América Latina y Chile. Santiago, Chile: LOM.

González, J. (2009). Ciudadanía juvenil en el Chile post-dictadura. El movimiento secundario del año 2006 y las organizaciones de autoeducación popular en Santiago de Chile. En OPECH. Juventud y Enseñanza Media en Chile del Bicentenario. Antecedentes de la Revolución Pingüina (pp. 394-432). Santiago, Chile: Universidad de Chile. 
González, J. y Sánchez, R. (Ed.). (2012). 2011: Aportes para interpretar una década de lucha por autoeducación. Santiago, Chile: Quimantú.

Grez, S. (2011). Un nuevo amanecer de los movimientos sociales. Revista The Clinic 409. Recuperado de www.theclinic.cl

Grez, S. (2012). Chile 2012: el movimiento estudiantil en la encrucijada. Le Monde Diplomatique Enero-febrero 2012, pp. 6-7.

Iglesias, M. (2011). Teoría en movimiento: más de una década de pensamiento crítico. Revista OSAL Año XII, N³0, pp. 25-43.

Mayol, A. (2012). El fantasma de la democracia. En VV.AA. Análisis del año 2011. Santiago: Departamento de Sociología, U. de Chile, pp. 81-90.

Núñez, D. (2011). Proyecciones políticas del movimiento social por la educación en Chile. Revista OSAL Año XIII, N 31, pp. 61-70.

OPECH (2010). De actores secundarios a estudiantes protagonistas. Versión 2.0. Santiago, Chile: Quimantú.

Pérez Ledesma, M. (1994). Cuando lleguen los días de cólera (Movimientos sociales, teoría e historia). Revista Zona Abierta $\mathrm{N}^{\circ}$ 69, pp. 51-120.

Ramis, A. (2009). ¿Cómo salir de la jaula de hierro constitucional?. Recuperado de: $\quad$ http://alvaroramis.wordpress.com/2009/10/01/\%C2\%BFcomosalir-de-la-jaula-de-hierro-constitucional

Ruiz, C. y Sáez, B. (2012). La irrupción de los hijos de la modernización. En VV.AA.: Análisis del año 2011. Santiago: Departamento de Sociología, U. de Chile, pp. 27-44.

Salazar, G. (2012). Movimientos sociales en Chile. Trayectoria histórica y proyección política. Santiago, Santiago: Uqbar.

Zibechi, R. (2008). Autonomías y emancipaciones. América Latina en movimiento. Santiago, Chile: Quimantú.

Zibechi, R. (2011). El pensamiento crítico en el laberinto del progresismo. Revista OSAL Año XII, N³0, pp. 19-24.

Zibechi, R. (2011a). Cuando el presente deja de ser una extensión del pasado. En R. Gutiérrez (et. al). Palabras para tejernos, resistir y transformar en la época que estamos viviendo (pp. 57-76). Cochabamba: Textos Rebeldes.

FECHA DE RECEPCIÓN: MAYO 2018.

FECHA DE ACEPTACIÓN: MAYO 2018. 